\title{
Analysis of the functional polymorphism in the cytochrome P450 CYP2C8 gene rs11572080 with regard to colorectal cancer risk
}

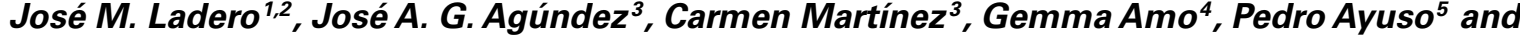 \\ Elena García-Martín ${ }^{4}$ \\ 1 Service of Gastroenterology, Hospital Clínico San Carlos, Instituto de Investigación Sanitaria del Hospital Clínico San Carlos, Madrid, Spain \\ ${ }^{2}$ Department of Medicine, Medical School, Universidad Complutense, Madrid, Spain \\ ${ }^{3}$ Department of Pharmacology, University of Extremadura, Cáceres, Spain \\ ${ }^{4}$ Department of Biochemistry and Molecular Biology, University of Extremadura, Cáceres, Spain \\ ${ }^{5}$ Fundación Instituto Mediterraneo Para el Avance en Biotecnología e Investigación Sanitaria, Málaga, Spain
}

\section{Edited by:}

Kathrin Klein, Dr. Margarete Fischer-Bosch-Institute of Clinical Pharmacology, Germany

\section{Reviewed by:}

Rheem Totah, University of Washington, USA

Rajiv Kumar, German Cancer

Research Center, Germany

\section{*Correspondence:}

Elena García-Martín, Departamento de Bioquímica y Biología Molecular y Genética, Universidad de Extremadura, Avda de la Universidad s/n 10071-Cáceres, Spain. e-mail: elenag@unex.es
In addition to the known effects on drug metabolism and response, functional polymorphisms of genes coding for xenobiotic-metabolizing enzymes (XME) play a role in cancer. Genes coding for XME act as low-penetrance genes and confer modest but consistent and significant risks for a variety of cancers related to the interaction of environmental and genetic factors. Consistent evidence supports a role for polymorphisms of the cytochrome P450 CYP2C9 gene as a protecting factor for colorectal cancer susceptibility. It has been shown that CYP2C8 and CYP2C9 overlap in substrate specificity. Because CYP2C8 has the common functional polymorphisms rs 11572080 and rs10509681 (CYP2C8*3), it could be speculated that part of the findings attributed to CYP2C9 polymorphisms may actually be related to the presence of polymorphisms in the CYP2C8 gene. Nevertheless, little attention has been paid to the role of the CYP2C8 polymorphism in colorectal cancer. We analyzed the influence of the CYP2C8*3 allele in the risk of developing colorectal cancer in genomic DNA from 153 individuals suffering colorectal cancer and from 298 age- and gender-matched control subjects. Our findings do not support any effect of the CYP2C $8^{*} 3$ allele (OR for carriers of functional CYP2C8 alleles $=0.50(95 \% \mathrm{Cl}=0.16-1.59 ; p=0.233)$. The absence of a relative risk related to $C Y P 2 C 8^{*} 3$ did not vary depending on the tumor site. We conclude that the risk of developing colorectal cancer does not seem to be related to the commonest functional genetic variation in the CYP2C8 gene.

Keywords: CYP2C8, colorectal cancer risk, polymorphisms, xenobiotic-metabolizing enzymes, biomarkers

\section{INTRODUCTION}

In addition to the known effects on drug metabolism and response, functional polymorphisms of genes coding for xenobioticmetabolizing enzymes (XME) play a modest but consistent role in cancer risk. Genes coding for XME act as low-penetrance genes and confer significant risks for a variety of cancers related to the interaction of environmental and genetic factors (Agundez, 2004). Cytochrome P450 (CYP) enzymes are involved in the metabolism of drugs and other xenobiotics. Two enzymes of the CYP2C subfamily, CYP2C8 and CYP2C9, have received special attention because they are involved in the metabolism of several commonly used drugs and are capable of activating carcinogens and mutagens. Consistent evidence supports a role for polymorphisms of the cytochrome P450 CYP2C9 gene that produce decreased enzymatic activity as a protecting factor for colorectal cancer susceptibility (Martinez et al., 2001; Chan et al., 2004, 2009; Cleary et al., 2010; Northwood et al., 2010).

One of the mechanisms proposed for this protecting effect is based on the effect of non-steroidal anti-inflammatory drugs (NSAIDs). Inflammation is a known risk factor for colorectal cancer, and the use of NSAIDs constitute a potential means of decreasing inflammation in the colonic epithelium (Ulrich et al., 2006). For this reason, interindividual variability in the metabolism of NSAIDs may play a role in colorectal cancer risk (Bosetti et al., 2012). Two cytochrome P450 enzymes, namely CYP2C9 and, to a lesser extent, CYP2C8, play a major role in the metabolism of NSAIDs (for a review, see Agundez et al., 2009). CYP2C enzymes are expressed in human colon and in colorectal cancer cells (Martinez et al., 2002; Bergheim et al., 2005b; Garcia-Martin et al., 2006b). In addition CYP2C8 expression in colorectal cancer cells is inducible (Garcia-Martin et al., 2006b), and it has been postulated that altered expression of CYP2C enzymes might contribute to the development of colon cancer (Bergheim et al., 2005a).

Although it is known that CYP2C9 y CYP2C8 have common substrates including NSAIDs (Garcia-Martin et al., 2004; Martinez et al., 2005; Totah and Rettie, 2005), a putative association between CYP2C8 polymorphisms and colorectal cancer risk has not been analyzed in detail. Only the effect of CYP2C8 polymorphisms as modifying factors for the protective effect of regular NSAID use has been analyzed (McGreavey et al., 2005). This study 
indicated a lack of association of CYP2C8 polymorphisms, but also of CYP2C9 polymorphisms as modifiers of the protective effect of regular NSAID use on the risk of colorectal carcinoma. Nevertheless, the potential of CYP2C8 polymorphisms as potential modifiers of colorectal cancer risk remains to be analyzed in detail.

In the present study we analyzed the association of $C Y P 2 C 8^{*} 3$ with colorectal cancer in a Spanish population. CYP2C8 is involved in the metabolism of arachidonic and retinoic acid. It is the main enzyme involved in the metabolism of R-ibuprofen and it has been shown to make a significant contribution to the metabolism of many other NSAIDs (Martinez et al., 2006). Several variant alleles have been described for the CYP2C8 gene, CYP2C $8^{*} 3$ being the most common variant allele among Caucasian individuals (Garcia-Martin et al., 2006a). The effect in vivo of this mutation is a decrease in the metabolism and altered pharmacokinetics of CYP2C8 substrates (Martinez et al., 2005).

\section{MATERIALS AND METHODS}

The study group consisted of 153 unrelated consecutive patients (82 men and 71 women) with colorectal cancer, and 298 healthy subjects (Table 1). These subjects have participated in previous studies (Garcia-Martin et al., 2001; Martinez et al., 2001). All the participants were white Spanish individuals, living in the same areas as the patients (Madrid and surrounding areas), and were included in the study after giving informed written consent. The diagnosis was based on histology analyses of endoscopic biopsies and/or surgical resection specimens. Data regarding known previous digestive diseases, alcohol, and tobacco consumption, serum tests for hepatitis B and C virus and other diseases were collected. Heavy drinkers were defined as individuals drinking more than $50 \mathrm{~g}$ of alcohol per day. All the patients were requested to participate in the study, and all of them agreed to do so. Control samples were obtained from medical students, University, and Hospital staff. A medical examination was carried out to identify subjects in good health. Over 95\% of the healthy subjects requested agreed to participate in the study. The protocol was approved by the Ethics Committee of the San Carlos University Hospital, Madrid. A possible confounding factor in the present study is that, within a study group, the frequency of individuals carrying a determined variant allele may change with age in the event that the presence of such an allele would be related to severe diseases. If a determined genotype has a "protecting effect" against any disease, it may be expected that in populations consisting of older subjects there is an increased frequency of such a protecting genotype. Therefore we included within the control group a selected subgroup of 41 healthy subjects with ages ranging from 90 to 95 years (Martinez et al., 2001). Genotype analysis indicates frequencies that were identical to those of younger healthy subjects (see Results). Another possible confounder is related to the fact that the control subjects are highly educated people, and differences in lifestyle as compared to cancer patients may be expected. Since digestive cancers are partly related to diet, these changes in lifestyle may be relevant. However, it should be stated that patients and controls were interviewed to assure that diet and lifestyle did not differ between patients and control subjects. Blood samples from all participants were stored at $-80^{\circ} \mathrm{C}$ until analysis and genomic DNA was prepared from peripheral leukocytes. The analyses for the $C Y P 2 C 8^{*} 3$ gene variants were carried out by amplification-restriction procedures. Briefly, the pair of primers $5^{\prime}$-CTTCCGTGCTACATGATGACG-3' $3^{\prime}$ and $5^{\prime}$-CTGCTGAGAAAGGCATGAAG-3' was used for a mismatch PCR-RFLP test. The $117 \mathrm{bp}$ PCR product was purified and digested with the endonuclease $X m n I$, that produced two fragments of 92 and $25 \mathrm{bp}$ in the $C Y P 2 C 8^{*} 1$ allele product, and did not digest the CYP2C8*3 PCR product. Further details are described elsewhere (Martinez et al., 2005). Twenty DNA samples with every genotype $\left(C Y P 2 C 8^{*} 1{ }^{*} 1\right.$ and $\left.{ }^{*} 1{ }^{*} 3\right)$ and all samples with the $C Y P 2 C 8^{*} 3 *^{*} 3$ genotypes were cross-tested with TaqMan probes designed to detect the CYP2C8*3 variant allele (rs11572080; C_25625794_10; Applied Biosystems, Madrid, Spain) and in all cases the genotypes obtained by amplification-restriction and by the TaqMan analyses fully corresponded.

\section{STATISTICAL ANALYSIS}

The intergroup comparison values were calculated using chisquare or Fisher's exact tests when appropriate. Logistic regression analyses were performed using the SPSS 15.0 for Windows (SPSS Inc., Chicago, IL, USA) to test for confounders, including gender, age at diagnosis, smoking and drinking habits, and tumor localization. The $95 \%$ confidence intervals were also calculated. The statistical power calculated for the sample size of this study, determined from allele frequencies with a genetic model analyzing the frequency of the risk gene with $\mathrm{RR}=2.0 ; p=0.05$ ) for two-tailed and one-tailed associations of the risk with the variant allele is 98.1 and $99.2 \%$, respectively. The minimum odds ratio detectable

Table 1 | Characteristics of the individuals included in the study.

\begin{tabular}{|c|c|c|c|c|}
\hline & \multicolumn{2}{|c|}{ Patients } & \multicolumn{2}{|c|}{ Controls } \\
\hline & Male $(n=82)$ & Female $(n=71)$ & Male $(n=160)$ & Female $(n=138)$ \\
\hline Age years (mean $\pm S D$ ) & $66.9 \pm 9.6$ & $65.6 \pm 12.9$ & $65.8 \pm 10.2$ & $66.0 \pm 11.7$ \\
\hline Non-smokers/smokers & $53 / 29$ & $69 / 2$ & $108 / 52$ & $129 / 9$ \\
\hline Non-drinkers/drinkers & $60 / 22$ & $71 / 0$ & $141 / 19$ & $138 / 0$ \\
\hline \multicolumn{5}{|l|}{ TUMOR SITE } \\
\hline Non-sigmoid & 33 & 22 & - & - \\
\hline Sigmoid & 26 & 22 & - & - \\
\hline Rectum & 23 & 27 & - & - \\
\hline
\end{tabular}


with this sample size is 1.55 , with a statistical power for one-tailed association equal to $80 \%$.

\section{RESULTS}

Table 2 shows the CYP2C $8^{*} 1 /^{*} 3$ genotypes and allele frequencies in colorectal cancer patients and healthy subjects. Differences noted by the comparison of genotype frequencies among them were not statistically significant. Odds ratio for carriers of the functional CYP2C $8^{*} 1$ allele $=0.50$ 95\% C.I. $=0.160-1.59 ; \chi^{2}=1.42$; $p=0.233$. These findings were not influenced by gender, previous surgical therapy, or chemotherapy and Dukes' stage of the tumor. Genotypes in both patients and control subjects are in HardyWeinberg's equilibrium. Deviation test from Hardy-Weinberg's equilibrium (Pearson): $p=0.172$ (patients); $p=0.265$ (controls). Trend test with the number of variant $C Y P 2 C 8^{*} 3$ alleles: $p=0.584$.

To elucidate whether the CYP2C8 genotype could be related to a particular type of colorectal cancer, patients were divided into three subgroups according to the anatomical site of the tumor (rectum, sigmoid colon, and non-sigmoid colon). Table 3 shows that none of the intergroup comparisons or comparisons with control subjects were significant. Deviation test from Hardy-Weinberg's equilibrium (Pearson): $p=0.603$ (non-sigmoid); $p=0.167$ (sigmoid); $p=0.587$ (rectum). Logistic regression analyses did not indicate any influence of confounders such as gender, age at diagnosis, smoking and drinking habits, or tumor localization in the negative findings obtained in this study.

\section{DISCUSSION}

The clinical use of genetic biomarkers for the identification of high risk subpopulations is a major goal which scientists have long been pursuing. In the case of colorectal cancer, the search for risk biomarkers by means of genome wide association studies (GWAs) has provided relevant information, pointing in particular to the single nucleotide polymorphism rs3802842 at 11q23.1 and five additional loci. When SNPs in these loci are combined, the risk increases with an increasing number of variant alleles (Pittman et al., 2008; Tomlinson et al., 2008). Nevertheless, the biomarkers identified in GWA studies explain only a minor percentage of the risk and so these studies should be completed with additional research.

Various candidate gene association studies (CGAs) have been carried out in an attempt to identify low-penetrance genes capable of increasing the risk of developing colorectal cancer. With regard to cytochrome P450 enzymes, two mechanisms have been proposed to explain a putative association between the CYP2C9 polymorphism and colorectal cancer risk. A number of xenobiotics that are ingested as pyrolysis products have been related to colorectal cancer risk. These include polycyclic aromatic hydrocarbons and heterocyclic aromatic amines. Several of these mutagenic substances are CYP2C substrates (Snyderwine et al., 1997; Jonsdottir et al., 2012) and therefore a genetically determined alteration in the metabolism of carcinogens and mutagens may underlie the observed association. This first hypothesis would imply that, besides CYPs, polymorphisms in other enzymes involved in the metabolism of polycyclic aromatic hydrocarbons and heterocyclic aromatic amines would influence the risk. This hypothesis is partly supported by the influence of NAT2 polymorphisms in colorectal cancer risk (revised in Agundez, 2008). The second mechanism proposed to explain the association between the CYP2C9 polymorphism and colorectal cancer risk is the key role that CYP2C9 plays in the metabolism of NSAIDs. This, together with the protective effect that NSAIDs play in human colorectal cancer, leads to the theory that in individuals with high CYP2C9 enzyme activity the protective effect of NSAIDs could be diminished. Because CYP2C8 and CYP2C9 show partial overlapping in substrate specificity, particularly related to some NSAIDs such as ibuprofen, celecoxib, diclofenac, or tenoxicam (Tang, 2003; Garcia-Martin et al., 2004; Martinez et al., 2005; Daly et al., 2007; Daily and Aquilante, 2009), it could be speculated that the presence of defect CYP2C8 variant alleles may influence the risk of developing colorectal cancer.

To date, only the effect of polymorphisms in CYP2C8 as modifiers of the protective effect of regular NSAID use has been analyzed. (McGreavey et al., 2005) In this study, CYP2C8 or CYP2C9 polymorphisms did not influence the protective effect of regular NSAID use on the risk of colorectal cancer. Because CYP2C8 and CYP2C9 polymorphisms show a great interethnic and intraethnic variability (Martinez et al., 2006) and because positive association of CYP2C9 genotypes with colorectal cancer risk was observed in some populations (Bigler et al., 2001; Martinez et al., 2001; Tranah et al., 2005; Hubner et al., 2006; Samowitz et al., 2006; Liao et al., 2007; Cotterchio et al., 2008; Cross et al., 2008; Chan et al., 2009; Siemes et al., 2009; Cleary et al., 2010) but not in other populations, and particularly in UK individuals (Sachse et al., 2002; Landi et al., 2005; Hubner et al., 2006; Kury et al., 2007). It could be speculated that the association of low-penetrance genes with colorectal cancer risk may vary from one population to another. Diet is a key

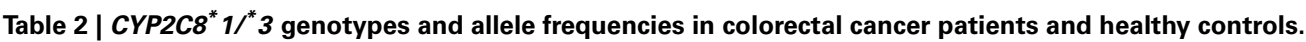

\begin{tabular}{|c|c|c|c|}
\hline & Patients ( $n=153,306$ alleles) & Controls ( $n=298,596$ alleles) & OR $(95 \% \mathrm{Cl}), p$ \\
\hline \multicolumn{4}{|c|}{ GENOTYPES rs $11572080 \mathrm{C} / \mathrm{T}(* 1 / * 3)$} \\
\hline CYP2C8*1/*1 & $111(72.5 ; 65.5-79.6)$ & $202(67.8 ; 62.5-73.1)$ & $1.26(0.80-1.98), 0.299$ \\
\hline CYP2C $8 * 1 /{ }^{*} 3$ & $36(23.5 ; 16.8-30.3)$ & $90(30.2 ; 25.0-35.4)$ & $0.71(0.44-1.14), 0.135$ \\
\hline $\mathrm{CYP} 2 \mathrm{C} 8 * 3 /{ }^{*} 3$ & $6(3.9 ; 0.8-7.0)$ & $6(2.0 ; 0.4-3.6)$ & $1.99(0.56-7.09), 0.233$ \\
\hline \multicolumn{4}{|c|}{ ALLELE FREQUENCIES } \\
\hline
\end{tabular}

The values in each cell represent: number (percentage) and [95\% confidence intervals]. 
Table 3 | CYP2C8* $1{ }^{*} 3$ genotypes of patients with colorectal cancer according to tumor site.

\begin{tabular}{|c|c|c|c|}
\hline & Non-sigmoid & Sigmoid & Rectum \\
\hline \multicolumn{4}{|c|}{ GENOTYPES rs11572080 C/T (*1/*3) } \\
\hline CYP2C8*1/*1 & 39 (70.9; 58.9-82.9) & 37 (77.1; 65.2-89.0) & $35(70.0 ; 57.3-82.7)$ \\
\hline CYP2C8*1/*3 & $14(25.5 ; 13.9-37.0)$ & 9 (18.8; 7.7-29.8) & $13(26.0 ; 13.8-38.2)$ \\
\hline$C Y P 2 C 8^{*} 3 /{ }^{*} 3$ & $2(3.6 ; 0-8.6)$ & $2(4.2 ; 0-9.8)$ & $2(4.0 ; 0-9.4)$ \\
\hline \multicolumn{4}{|l|}{ HAPLOTYPES } \\
\hline CYP2C8*1 & 92 (83.6; 76.7-90.5) & 83 (86.5; 79.6-93.3) & $83(83.0 ; 75.6-90.4)$ \\
\hline CYP2C8*3 & $18(16.4 ; 9.5-23.3)$ & $13(13.5 ; 6.7-20.4)$ & $17(17.0 ; 9.6-24.4)$ \\
\hline
\end{tabular}

The values in each cell represent: number (percentage) and (95\% confidence intervals).

factor in colorectal cancer risk, and because dietary determinants of colorectal cancer may vary greatly in different geographic locations, the contribution of low-penetrance genes to the overall risk may vary across populations.

For the above-mentioned reasons we decided to undertake the study in a population in which a positive effect of CYP2C9 polymorphism in colorectal cancer risk had already been identified. Because $C Y P 2 C 9^{*} 2$ and $C Y P 2 C 8^{*} 3$ variant alleles are at linkage disequilibrium (Yasar et al., 2002; Martinez et al., 2005, 2007), it could be expected that the association observed between the CYP2C9 genotypes and colorectal cancer risk (Martinez et al., 2001; Chan et al., 2004, 2009; Cleary et al., 2010; Northwood et al., 2010), could be partly related to the presence of the CYP2C8*3 variant allele. However, the findings obtained in the present study indicate that no major association of the $C Y P 2 C 8^{*} 3$ variant allele and colorectal cancer risk is present in the population analyzed.

A limiting factor in this study is that no other defect CYP2C8 alleles were analyzed. However, it should be emphasized that we tested for $C Y P 2 C 8^{*} 3$ which is the most common defect CYP2C8 allele in Caucasian individuals (see the website http://www.cypalleles.ki.se/cyp2c8.htm), whereas other variant

\section{REFERENCES}

Agundez, J. A. (2004). Cytochrome P450 gene polymorphism and cancer. Curr. Drug Metab. 5, 211-224.

Agundez, J. A. (2008). Polymorphisms of human $\mathrm{N}$-acetyltransferases and cancer risk. Curr. Drug Metab. 9, 520-531.

Agundez, J. A., Garcia-Martin, E., and Martinez, C. (2009). Genetically based impairment in CYP2C8and CYP2C9-dependent NSAID metabolism as a risk factor for gastrointestinal bleeding: is a combination of pharmacogenomics and metabolomics required to improve personalized medicine? Expert Opin. Drug Metab. Toxicol. 5, 607-620.

Bergheim, I., Bode, C., and Parlesak, A. (2005a). Decreased expression of cytochrome $\mathrm{P} 450$ protein in nonmalignant colonic tissue of patients with colonic adenoma. BMC Gastroenterol. 5:34. doi:10.1186/1471230X-5-34

Bergheim, I., Bode, C., and Parlesak, A. (2005b). Distribution of cytochrome P450 2C, 2E1, $3 \mathrm{~A} 4$, and $3 \mathrm{~A} 5$ in human colon mucosa. BMC Clin. Pharmacol. 5:4. doi:10.1186/1472-6904-5-4

Bigler, J., Whitton, J., Lampe, J. W., Fosdick, L., Bostick, R. M., and Potter, J. D. (2001). CYP2C9 and UGT1A6 genotypes modulate the protective effect of aspirin on colon adenoma risk. Cancer Res. 61, 3566-3569. J., and La Vecchia, C. (2012). Aspirin and cancer risk: a quantitative review to 2011. Ann. Oncol. 23, 1403-1415.

Chan, A. T., Tranah, G. J., Giovannucci, E. L., Hunter, D. J., and Fuchs, C. S. (2004). A prospective study of genetic polymorphisms in the cytochrome P-450 2C9 enzyme and
Bosetti, C., Rosato, V., Gallus, S., Cuzick,

alleles such as $C Y P 2 C 8^{*} 2, C Y P 2 C 8^{*} 4$, or $C Y P 2 C 8^{*} 5$ are extremely rare or no conclusive evidence for their deleterious effect on drug metabolism in vivo is available, revised in (Martinez et al., 2006). Another putative limiting factor in this study is the sample size. It should however be emphasized that in the study population the positive association of colorectal cancer risk with CYP2C9 genotype was identified with a smaller sample size (Martinez et al., 2001) and hence, in the event that a major linkage between CYP2C8* 3 and colorectal cancer risk might exist in the population studied, the sample size would be enough to identify it. In conclusion, our findings, with sufficient statistical power to detect an odds ratio equal to 1.6 , indicate that the $C Y P 2 C 8^{*} 3$ variant allele does not show a major association with colorectal cancer risk.

\section{ACKNOWLEDGMENTS}

FIS PS09/00943, PS09/00469, and RETICS RD07/0064/0016 from Fondo de Investigación Sanitaria, Instituto de Salud Carlos III, Spain, and GR10068 from Junta de Extremadura, Spain. Financed in part with FEDER funds from the European Union. We are grateful to Prof. James McCue for assistance in language editing, and to Ms. Gara Esguevillas for technical assistance.

the risk for distal colorectal adenoma. Clin. Gastroenterol. Hepatol. 2, 704-712.

Chan, A. T., Zauber, A. G., Hsu, M., Breazna, A., Hunter, D. J., Rosenstein, R. B., et al. (2009). Cytochrome P450 2C9 variants influence response to celecoxib for prevention of colorectal adenoma. Gastroenterology 136, 2127-2136.

Cleary, S. P., Cotterchio, M., Shi, E., Gallinger, S., and Harper, P. (2010). Cigarette smoking, genetic variants in carcinogen-metabolizing enzymes, and colorectal cancer risk. Am. J. Epidemiol. 172, 1000-1014.

Cotterchio, M., Boucher, B. A., Manno, M., Gallinger, S., Okey, A. B., and Harper, P. A. (2008). Red meat intake, doneness, polymorphisms in genes that encode carcinogenmetabolizing enzymes, and colorectal cancer risk. Cancer Epidemiol. Biomarkers Prev. 17, 3098-3107.
Cross, J. T., Poole, E. M., and Ulrich, C. M. (2008). A review of genedrug interactions for nonsteroidal anti-inflammatory drug use in preventing colorectal neoplasia. Pharmacogenomics J. 8, 237-247.

Daily, E. B., and Aquilante, C. L. (2009). Cytochrome $\mathrm{P} 4502 \mathrm{C} 8$ pharmacogenetics: a review of clinical studies. Pharmacogenomics 10, 1489-1510.

Daly, A. K., Aithal, G. P., Leathart, J. B., Swainsbury, R. A., Dang, T. S., and Day, C. P. (2007). Genetic susceptibility to diclofenac-induced hepatotoxicity: contribution of UGT2B7, CYP2C8, and ABCC2 genotypes. Gastroenterology 132, 272-281.

Garcia-Martin, E., Martinez, C., Ladero, J. M., and Agundez, J. A. (2006a). Interethnic and intraethnic variability of CYP2C8 and CYP2C9 polymorphisms in healthy individuals. Mol. Diagn. Ther. 10, 29-40. 
Garcia-Martin, E., Pizarro, R. M., Martinez, C., Gutierrez-Martin, Y., Perez, G., Jover, R., et al. (2006b). Acquired resistance to the anticancer drug paclitaxel is associated with induction of cytochrome P450 2C8. Pharmacogenomics 7, 575-585.

Garcia-Martin, E., Martinez, C., Ladero, J. M., Gamito, F. J., and Agundez, J. A. (2001). High frequency of mutations related to impaired CYP2C9 metabolism in a Caucasian population. Eur. J. Clin. Pharmacol. 57, 47-49.

Garcia-Martin, E., Martinez, C. Tabares, B., Frias, J., and Agundez, J. A. (2004). Interindividual variability in ibuprofen pharmacokinetics is related to interaction of cytochrome P450 2C8 and 2C9 amino acid polymorphisms. Clin. Pharmacol. Ther. 76, 119-127.

Hubner, R. A., Muir, K. R., Liu, J. F., Logan, R. F., Grainge, M., Armitage, N., et al. (2006). Genetic variants of UGT1A6 influence risk of colorectal adenoma recurrence. Clin. Cancer Res. 12, 6585-6589.

Jonsdottir, S. O., Ringsted, T., Nikolov, N. G., Dybdahl, M., Wedebye, E. B., and Niemela, J. R. (2012). Identification of cytochrome P450 2D6 and 2C9 substrates and inhibitors by QSAR analysis. Bioorg. Med. Chem. 20, 2042-2053.

Kury, S., Buecher, B., Robiou-Du-Pont, S., Scoul, C., Sebille, V., Colman, H., et al. (2007). Combinations of cytochrome P450 gene polymorphisms enhancing the risk for sporadic colorectal cancer related to red meat consumption. Cancer Epidemiol. Biomarkers Prev. 16, 1460-1467.

Landi, S., Gemignani, F., Moreno, V., Gioia-Patricola, L., Chabrier, A., Guino, E., et al. (2005). A comprehensive analysis of phase I and phase II metabolism gene polymorphisms and risk of colorectal cancer. Pharmacogenet. Genomics 15, 535-546.

Liao, L. H., Zhang, H., Lai, M. P., Lau, K. W., Lai, A. K., Zhang, J. H., et al. (2007). The association of CYP2C9 gene polymorphisms with colorectal carcinoma in Han Chinese. Clin. Chim. Acta 380, 191-196.

Martinez, C., Blanco, G., Garcia-Martin, E., and Agundez, J. A. (2006). Clinical pharmacogenomics for CYP2C8 and CYP2C9: general concepts and application to the use of NSAIDs. Farm. Hosp. 30, 240-248.

Martinez, C., Garcia-Martin, E., AlonsoNavarro, H., Jimenez-Jimenez, F. J., Benito-Leon, J., Garcia-Ferrer, I., et al. (2007). Changes at the CYP2C locus and disruption of CYP2C8/9 linkage disequilibrium in patients with essential tremor. Neuromolecular Med. 9, 195-204.

Martinez, C., Garcia-Martin, E., Blanco, G., Gamito, F. J., Ladero, J. M., and Agundez, J. A. (2005). The effect of the cytochrome P450 CYP2C8 polymorphism on the disposition of (R)ibuprofen enantiomer in healthy subjects. Br. J. Clin. Pharmacol. 59, 62-69.

Martinez, C., Garcia-Martin, E., Ladero, J. M., Sastre, J., Garcia-Gamito, F., Diaz-Rubio, M., et al. (2001). Association of CYP2C9 genotypes leading to high enzyme activity and colorectal cancer risk. Carcinogenesis 22, 1323-1326.

Martinez, C., Garcia-Martin, E., Pizarro, R. M., Garcia-Gamito, F. J., and Agundez, J. A. (2002). Expression of paclitaxel-inactivating CYP3A activity in human colorectal cancer: implications for drug therapy. $\mathrm{Br}$. J. Cancer 87, 681-686.

McGreavey, L. E., Turner, F., Smith, G., Boylan, K., Timothy Bishop, D., Forman, D., et al. (2005). No evidence that polymorphisms in CYP2C8, CYP2C9, UGT1A6, PPARdelta and PPARgamma act as modifiers of the protective effect of regular NSAID use on the risk of colorectal carcinoma. Pharmacogenet. Genomics 15, 713-721.

Northwood, E. L., Elliott, F., Forman, D., Barrett, J. H., Wilkie, M. J., Carey, F. A., et al. (2010). Polymorphisms in xenobiotic metabolizing enzymes and diet influence colorectal adenoma risk. Pharmacogenet. Genomics 20, 315-326.

Pittman, A. M., Webb, E., CarvajalCarmona, L., Howarth, K., D Bernardo, M. C., Broderick, P., et al. (2008). Refinement of the basis and impact of common 11q23.1 variation to the risk of developing colorectal cancer. Hum. Mol. Genet. 17, 3720-3727.

Sachse, C., Smith, G., Wilkie, M. J., Barrett, J. H., Waxman, R., Sullivan, F., et al. (2002). A pharmacogenetic study to investigate the role of dietary carcinogens in the etiology of colorectal cancer. Carcinogenesis 23 , 1839-1849.

Samowitz, W. S., Wolff, R. K., Curtin, K., Sweeney, C., Ma, K. N., Andersen, K., et al. (2006). Interactions between CYP2C9 and UGT1A6 polymorphisms and nonsteroidal anti-inflammatory drugs in colorectal cancer prevention. Clin. Gastroenterol. Hepatol. 4, 894-901.

Siemes, C., Eijgelsheim, M., Dieleman, J. P., Van Schaik, R. H., Uitterlinden, A. G., Van Duijn, C. M., et al. (2009). No modification of the beneficial effect of NSAIDs on colorectal cancer by CYP2C9 genotype. Neth. J. Med. 67, 134-141.

Snyderwine, E. G., Turesky, R. J. Turteltaub, K. W., Davis, C. D. Sadrieh, N., Schut, H. A., et al. (1997). Metabolism of food-derived heterocyclic amines in nonhuman primates. Mutat. Res. 376, 203-210.

Tang, W. (2003). The metabolism of diclofenac - enzymology and toxicology perspectives. Curr. Drug Metab. 4, 319-329.

Tomlinson, I. P., Webb, E., CarvajalCarmona, L., Broderick, P., Howarth, K., Pittman, A. M., et al. (2008). A genome-wide association study identifies colorectal cancer susceptibility loci on chromosomes $10 \mathrm{p} 14$ and 8q23.3. Nat. Genet. 40, 623-630.

Totah, R. A., and Rettie, A. E. (2005). Cytochrome P450 2C8: substrates, inhibitors, pharmacogenetics, and clinical relevance. Clin. Pharmacol. Ther. 77, 341-352.
Tranah, G. J., Chan, A. T., Giovannucci, E., Ma, J., Fuchs, C., and Hunter D. J. (2005). Epoxide hydrolase and CYP2C9 polymorphisms, cigarette smoking, and risk of colorectal carcinoma in the nurses' health study and the physicians' health study. Mol. Carcinog. 44, 21-30.

Ulrich, C. M., Bigler, J., and Potter, J. D. (2006). Non-steroidal antiinflammatory drugs for cancer prevention: promise, perils and pharmacogenetics. Nat. Rev. Cancer 6 , 130-140.

Yasar, U., Lundgren, S., Eliasson, E., Bennet, A., Wiman, B., De Faire, U., et al. (2002). Linkage between the CYP2C8 and CYP2C9 genetic polymorphisms. Biochem. Biophys. Res. Commun. 299, 25-28.

Conflict of Interest Statement: The authors declare that the research was conducted in the absence of any commercial or financial relationships that could be construed as a potential conflict of interest.

Received: 16 October 2012; accepted: 15 November 2012; published online: 03 December 2012.

Citation: Ladero JM, Agúndez JAG, Martínez C, Amo G, Ayuso $P$ and Garcia-Martín E (2012) Analysis of the functional polymorphism in the cytochrome P450 CYP2C8 gene rs11572080 with regard to colorectal cancer risk. Front. Gene. 3:278. doi: 10.3389/fgene. 2012.00278

This article was submitted to Frontiers in Pharmacogenetics and Pharmacogenomics, a specialty of Frontiers in Genetics.

Copyright (c) 2012 Ladero, Agúndez, Martínez, Amo, Ayuso and GarcíaMartin. This is an open-access article distributed under the terms of the Creative Commons Attribution License, which permits use, distribution and reproduction in other forums, provided the original authors and source are credited and subject to any copyright notices concerning any third-party graphics etc. 